\title{
The Effect of Potato Varieties and Processing Methods on Glycemic Response
}

\author{
Reena Grittle Pinhero1, Qiang Liu², J. Alan Sullivan3, Massimo Marcone1, Rickey Y. Yada ${ }^{1,4^{*}}$ \\ ${ }^{1}$ Department of Food Science, University of Guelph, Guelph, Canada \\ ${ }^{2}$ Guelph Research and Development Centre, Agriculture and Agri-Food Canada, Guelph, Canada \\ ${ }^{3}$ Department of Plant Agriculture, University of Guelph, Guelph, Canada \\ ${ }^{4}$ Faculty of Land and Food Systems, University of British Columbia, Vancouver, Canada \\ Email: *r.yada@ubc.ca
}

How to cite this paper: Pinhero, R.G., Liu, Q., Sullivan, J.A., Marcone, M. and Yada, R.Y. (2020) The Effect of Potato Varieties and Processing Methods on Glycemic Response. American Journal of Plant Sciences, 11, 1144-1162.

https://doi.org/10.4236/ajps.2020.117081

Received: May 19, 2020

Accepted: July 26, 2020

Published: July 29, 2020

Copyright (C) 2020 by author(s) and Scientific Research Publishing Inc. This work is licensed under the Creative Commons Attribution International License (CC BY 4.0).

http://creativecommons.org/licenses/by/4.0/

cc) (i) Open Access

\begin{abstract}
In order to identify those potatoes which exert a low glycemic impact after processing, eight early potato varieties and four processing methods were evaluated for their total starch content, amylose content, rapidly digestible starch (RDS), slowly digestible starch (SDS), and resistant starch (RS), estimated glycemic index (eGI), glycemic load (eGL) as well as their relationship among each other. While all these profiles were highly dependent on the potato variety and processing methods, all the eight varieties were classified as low GL foods ( $\mathrm{p} \leq 0.05)$. A strong positive correlation was observed between eGI and RDS ( $r=0.84,0.79$, and 0.74) for retrograded and reheated, baked and microwaved varieties, respectively), whereas a moderate negative correlation was observed between eGI and RS for retrograded and reheated $(\mathrm{r}=$ $-0.39)$ and microwaved $(-0.37)$ varieties $(\mathrm{p} \leq 0.05)$. On the basis of these findings, it can be concluded that potato variety, processing methods, and starch characteristics define the eGI and eGL. Furthermore, for the varieties examined, the present study identified RDS as a major starch factor contributing to eGI.
\end{abstract}

\section{Keywords}

Potato, Rapidly Digestible Starch, Slowly Digestible Starch, Resistant Starch, Glycemic Index, Glycemic Load

\section{Introduction}

Potato, the third largest food crop worldwide following rice and wheat, is becoming ever more important as a food source given that it produces more dry matter and protein per hectare compared to major cereal crops. Potatoes, are al- 
so a readily assimilated source of carbohydrate energy [1]. Nutritionally, the role of carbohydrate metabolism is critical to the regulation of our energy intake and maintenance of body weight. The glycemic index (GI) is a very complex mathematical measure that allows carbohydrate foods to be classified on a scale of 0 100 based on their effects on postprandial blood glucose levels and can be defined as either low $(\leq 55)$, medium (56 - 69), or high $(\geq 70)$ [2]. High GI foods, due to their rapid and exaggerated effects on postprandial blood glucose and insulin levels, are associated with significant health implications such as obesity, glucose intolerance, leading to metabolic syndrome, and type 2 diabetes [3]. Several studies reported that potatoes generally have medium to high GI, which has often adversely affected their consumption, but have overlooked the many nutritional and health benefits of potatoes [4] [5]. GI varies depending on the potato variety, origin, and maturity while processing methods can alter starch digestibility, along with the foods that they are consumed with. Given that the clinical evaluation of GI is expensive, in vitro starch digestibility methods have been developed and validated with clinical method for use as an effective, quick, low cost alternative for the identification of low GI potatoes [6]. Ek et al. [6] reported a strong positive correlation between in vivo and in vitro GI methods, particularly in the case of in vitro starch hydrolysis of cooked potatoes at 120 min. In addition to GI, the amount of carbohydrate consumed per meal also affects blood glucose levels and insulin responses. Hence, the concept of glycemic load (GL) was developed to simultaneously describe the quality (GI) and quantity of carbohydrate in a meal or diet [7] [8] [9].

Potato starch consists of $70 \%-80 \%$ amylopectin which is a highly branched, high molecular weight, biopolymer. Amylose represents approximately $20 \%$ $30 \%$ of starch and is a relatively long, linear, $\alpha$-glucan with only a few branches [10] [11] [12]. The relative proportion of amylose and amylopectin is important given that amylose acts as a restraint to swelling and, upon cooling, forms retrograded starch more readily. It has been reported that the blood glucose responses of biscuits containing a greater proportion of amylose resulted in a lower glycemic response than those which contain a greater proportion of amylopectin [13]. Based on in vitro methods, starch can be classified into the nutritionally important starch fractions: rapidly digestible starch (RDS), slowly digestible starch (SDS), and resistant starch (RS) [14]. RDS is identified as the amount of starch that is hydrolyzed within the first $20 \mathrm{~min}$ after incubation with $\alpha$-amylase, which is responsible for raising blood glucose levels quickly. SDS is defined as the amount of starch hydrolyzed between 20 - 120 min while RS as the amount of starch that is not hydrolyzed after $120 \mathrm{~min}$ post incubation and is calculated by subtracting RDS and SDS from the total starch measured. RS, classified as dietary fiber, will reach the large intestine, where it is fermented, and is reported to have physiological effects such as being implicated in the prevention of colon cancer and cardiovascular disease as well as being important for maintaining gastrointestinal health [15]. In vitro starch digestibility assays have been shown to be a good predictor of the in vivo glycemic response of starchy foods [16]. Die- 
tary fiber has an impact on food by reducing the rate of glucose breakdown and absorption, thereby reducing excess glucose build up and regulating carbohydrate metabolism.

Besides total starch, structure, and moisture content [17], other factors, such as the maturity of the potato variety, growing conditions as well as cooking methods [18] can also impact the glycemic response. Table potatoes can be classified as early maturing (65 - 90 days), mid-season (90 - 120 days) and main crop varieties (>120 days) based on the duration of maturity. Early maturing potato varieties usually have a lower dry matter, higher moisture content and different starch structure [19]. Our previous study, focusing on early potatoes, have shown that the variety, starch structure, as well as digestibility and cooking processes, did in fact have an impact on the GI and GL [20]. The present study was conducted to include further processing methods which are commonly used for the identification and recommendation of potatoes in order to recommend the varieties and processing methods that may reduce GI and result in a low glycemic response.

\section{Materials and Methods}

\subsection{Potato Samples}

A total of 8 potato varieties (Adora, Yellow Star, Carlingford, Purple Fiesta, French Fingerlings, Ciklamen, Red Thumb, and Smart) were tested. Our previous study [20] evaluated 14 early potato varieties as fresh (uncooked), (2) after boiling (cooked) and (3) boiled and cooled for $48 \mathrm{~h}$ at $4^{\circ} \mathrm{C}$ (retrograded). Based on the results of estimated glycemic index and glycemic load of these varieties, we selected the eight varieties, under the processing conditions, which we considered the best performers in our previous research to include in this study. These varieties were collected from Grand Bend Produce, 10026 Walker Rd, Grand Bend, Ontario, Canada.

\subsection{Sample Preparation}

Retrograded samples were prepared by cutting potatoes (with the skin) into four quarters, boiling for 10 minutes in water (until the sample could be pierced through using a fork), and was refrigerated at $4^{\circ} \mathrm{C}$ for $48 \mathrm{~h}$. Retrograded and reheated samples were prepared by heating the retrograded samples for one minute in the microwave (Master Chef, $0.7 \mathrm{Cu} . \mathrm{ft}, 700$ Watts). Baked samples were prepared by baking the potato in a pre-heated oven at $350^{\circ} \mathrm{F}$ for 20 minutes until the potatoes were soft and the center could be easily punctured with a fork. Microwaved samples were prepared by cooking the quartered samples in a microwave with $25 \mathrm{ml}$ of water, on cook mode, for $5 \mathrm{~min}$ (Master Chef, $0.7 \mathrm{Cu} . \mathrm{ft}$, 700 Watts). One hundred grams of each of these samples were freeze-dried using a VIRTIS freeze drier (The Virtis Company, Gardiner, New York 12525). The freeze-dried samples were ground using a coffee grinder, passed through a 250 $\mu \mathrm{m}$ sieve, and kept in air-tight plastic bags at room temperature until the day of 
analysis.

\subsection{Moisture Content of Potato Tuber}

The moisture content of the potato samples was determined from the formula after freeze drying [(Fresh wt. - dry wt. $) /$ fresh wt.] $\times 100$. Moisture content of the freeze-dried samples was also determined by drying $2 \mathrm{~g}$ of sample in the oven at $130^{\circ} \mathrm{C}$, arriving at a constant weight. Total moisture content was determined by adding these two moisture contents [20].

\subsection{Total Starch (TS) Content of Potato Dry Matter}

TS content of potato dry matter, as prepared in Section 2.2, was determined using the Megazyme Total Starch method (AACC Method 76.13) as per the manufacturer's procedure (Megazyme International Ireland, Bray Business Park, Bray, Co. Wicklow, Ireland [20].

\subsection{Apparent Amylose Content of Potato Dry Matter}

The apparent amylose content in potato dry matter was determined by iodine colorimetry [21] using a UV/vis spectrophotometer by measuring the absorbance of the amylose-iodine complex at $625 \mathrm{~nm}$ [20].

\subsection{Thermal Properties}

The thermal properties of potato dry matter were measured using a differential scanning calorimeter (DSC) (TA Instruments 2920 Modulated DSC, New Castle, DE). Potato dry matter (12 mg, dry weight, obtained by freeze drying) were weighed into a high volume pan with $28 \mu \mathrm{L}$ of distilled water to give a moisture content of $70 \%$. The sample pan was sealed, equilibrated to room temperature for $2 \mathrm{~h}$, and then heated from $5^{\circ} \mathrm{C}$ to $180^{\circ} \mathrm{C}$ at a heating rate of $10^{\circ} \mathrm{C} / \mathrm{min}$. After initial heating to $180^{\circ} \mathrm{C}$, the sample pan was cooled to $5^{\circ} \mathrm{C}$. Once the temperature reached $5^{\circ} \mathrm{C}$, the sample was immediately removed from the DSC and stored at $4^{\circ} \mathrm{C}$ for 14 days. After this, the stored sample pan was again heated from $5^{\circ} \mathrm{C}$ to $180^{\circ} \mathrm{C}$ at a rate of $10^{\circ} \mathrm{C} / \mathrm{min}$ to measure the properties of starch retrogradation. The instrument was calibrated using indium and an empty pan was used as a reference. The onset $\left(T_{o}\right)$, peak $\left(T_{p}\right)$, and conclusion $\left(T_{c}\right)$ temperature, $s$ as well as enthalpy $(\Delta \mathrm{H})$ of endothermic transition, were determined from the DSC thermograms. Mean values of the duplicated measurements were calculated and reported [22].

\subsection{In Vitro Starch Digestibility of Processed Potatoes}

In vitro starch digestibility of retrograded, baked, microwaved and reheated potato samples was determined as per the method of Englyst et al. [14] with modifications after starch hydrolysis [23]. Percentages of RDS and SDS were calculated by using the amount of starch digested at $20 \mathrm{~min}$, and between 20 and 120 min, respectively, whereas the amount of RS was calculated by using the equa- 
tion $\mathrm{RS}=\mathrm{TS}-(\mathrm{RDS}+\mathrm{SDS})[20]$.

\section{8. eGI and eGL Determination}

Starch hydrolysis of retrograded, baked, microwaved and reheated potato samples was carried out for $0-120 \mathrm{~min}$ as described by Goni, Garcia-Alonso, Saura-Calixto [24] with some modifications. A nonlinear first-order equation,

$$
C=C_{\infty}\left(1-\mathrm{e}^{-k t}\right)
$$

which was established by Goni, Garcia-Alonso, Saura-Calixto [24], was used to describe the kinetics of hydrolysis of the potato samples. $C$ is the starch hydrolyzed at time $t, C_{\infty}$ is the equilibrium concentration at the final time (120 min); $k$ is the kinetic constant; and $t$ is the chosen time. The hydrolysis index (HI) was obtained by dividing the area under the hydrolysis curve (AUC) of the samples using white bread as a reference sample, as reported by Goni et al. [24]. The AUC was calculated by the equation

$$
\mathrm{AUC}=C_{\infty}\left(t_{f}-t_{o}\right)-\left(C_{\infty} \div k\right)\left(1-\mathrm{e}^{-K}\right)\left(t_{f}-t_{o}\right)
$$

where $t_{f}$ is the final time and $t_{o}$ is the initial time. The eGI was calculated from the equation

$$
\mathrm{eGI}=8.198+(0.862 \times \mathrm{HI})
$$

as described by Granfeldt [25]. The eGL for $100 \mathrm{~g}$ of the boiled and retrograded potato was calculated using the formula,

$$
\mathrm{eGL}=(\mathrm{eGI} \times \mathrm{ACH}) \div 100
$$

(http://www.glycemicindex.com/faqsList.php\#1) [20].

\subsection{Data Analysis}

Data analysis was carried out using GraphPad Prism 6 Software (GraphPad Software Inc., California, USA). Differences among the various treatments were carried out using one-way analysis of variance (ANOVA) while Tukey's multiple comparison test was used to compare the means $(\mathrm{p} \leq 0.05)$. Pearson correlation was also used to establish a relationship between eGI and the various, nutritionally important, starch fractions.

\section{Results and Discussion}

Potatoes are a good source of readily available carbohydrate, protein, as well as many antioxidants and minerals, especially potassium. However, it is also known as a medium to high GI food. Many recent studies have stressed the importance of GL, rather than GI, and how GI is affected by factors such as preparation as well as the foods consumed along with it. Our previous results [20] from the study of early potatoes treated with a limited number of processing methods identified that retrograded potatoes had a low eGI and eGL. In the present study, we included further processing methods which are commonly used for the treatment of potatoes in order to identify and recommend the varieties and 
processing methods that may further improve eGI and eGL.

\subsection{Moisture Content}

A significantly higher moisture content was observed in the Smart variety for all processing methods, followed by Carlingford and Yellow Star (Figure 1). The lowest moisture content was obtained in Purple Fiesta, Red Thumb, Ciklamen and French Fingerlings. The moisture content was lowest for the deep colored Purple Fiesta. It has been reported that the water content of different potato cultivars may exert a significant effect on their glycemic impact [17].

\subsection{Thermal Properties of Dry Matter}

The thermal properties, such as onset $\left(T_{o}\right)$, peak $\left(T_{p}\right)$, and conclusion $\left(T_{c}\right)$, as well as enthalpy, of dry matter obtained from retrograded, reheated, baked and microwaved potatoes in the presence of excess water during the initial heating and thermograms are provided in Table 1 and Figure 2(a). As shown in Figure 2 (a), the thermograms of freeze-dried retrograded potato dry matter from different varieties mainly exhibited a single endotherm transition. The other potato samples had similar thermogram profiles (Figure 2(a)). The single endotherm transition indicates that starch was not fully gelatinized during the processing

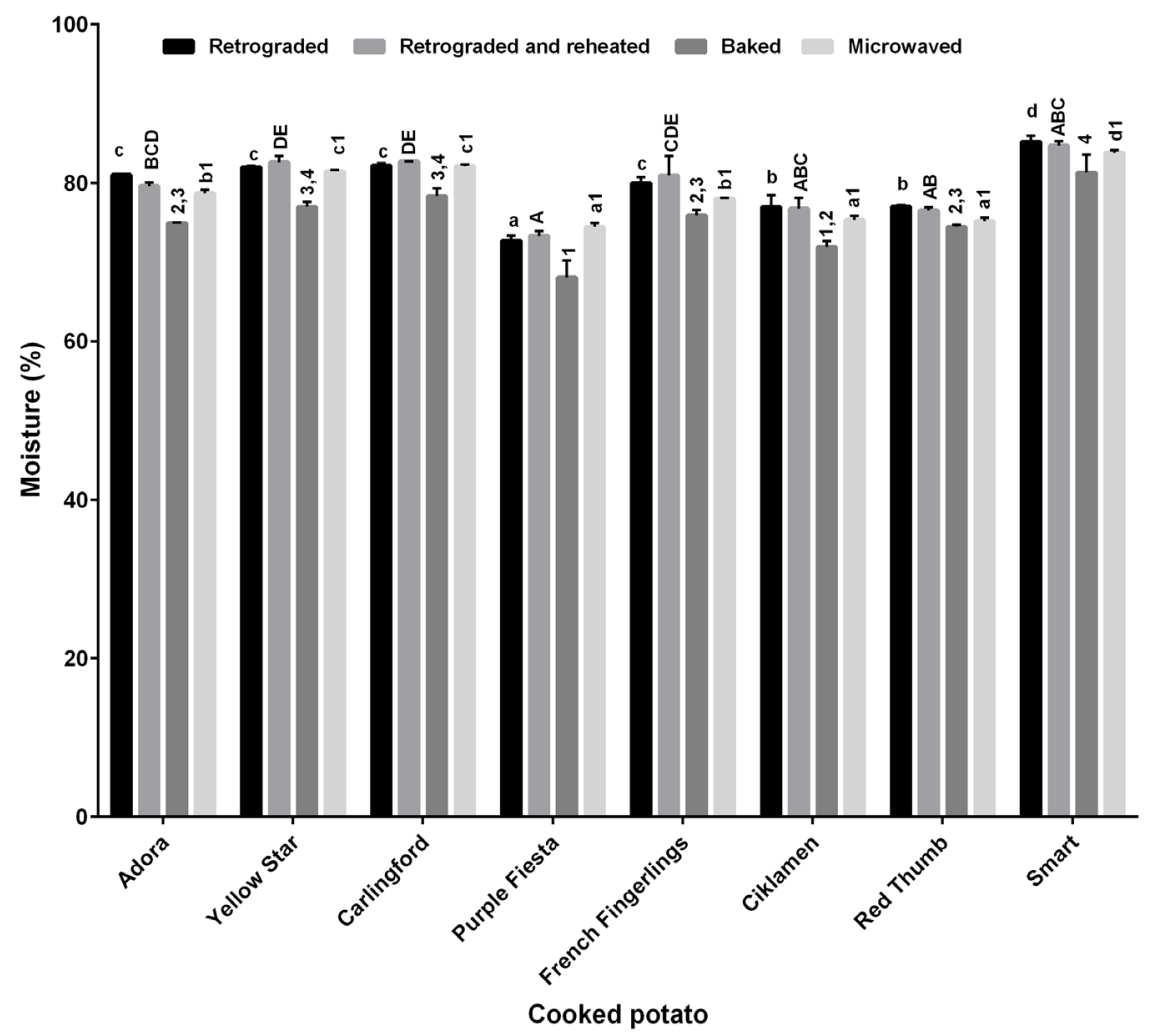

Figure 1. Effect of variety and processing on the moisture content of potatoes. Error bars represent standard error from two experiments. Bars with different letters, a - d, A - E, 1 4 and a1 - d1, denote significant differences between varieties when performing the same processing method (Tukey's test, $\mathrm{p} \leq 0.05$ ). 
Table 1. Gelatinization characteristics of potato dry matter.

\begin{tabular}{|c|c|c|c|c|c|}
\hline Cooking/Variety & $\begin{array}{c}\text { Onset } \\
\text { temperature } \\
T_{o}\left({ }^{\circ} \mathrm{C}\right)\end{array}$ & $\begin{array}{c}\text { Conclusion } \\
\text { temperature } \\
T_{c}\left({ }^{\circ} \mathrm{C}\right)\end{array}$ & $\begin{array}{c}T_{c}-T_{o} \\
\left({ }^{\circ} \mathrm{C}\right)\end{array}$ & $\begin{array}{c}\text { Peak } \\
\text { temperature } \\
T_{p}\left({ }^{\circ} \mathrm{C}\right)\end{array}$ & $\begin{array}{c}\text { Enthalpy } \\
\Delta \mathrm{H}\left(\mathrm{J} \cdot \mathrm{g}^{-1}\right)\end{array}$ \\
\hline \multicolumn{6}{|l|}{ Retrograded } \\
\hline Adora & 49 & 80.38 & 31.35 & 62.52 & 5.857 \\
\hline Yellow Star & 49.6 & 82.39 & 32.83 & 65.39 & 5.888 \\
\hline Carlingford & 51.1 & 81.97 & 30.84 & 66.1 & 5.672 \\
\hline Purple Fiesta & 48.7 & 81.04 & 32.35 & 64.38 & 6.175 \\
\hline French Fingerlings & 49.6 & 81.29 & 31.72 & 64.74 & 6.007 \\
\hline Ciklamen & 49.9 & 82.27 & 32.33 & 65.91 & 6.17 \\
\hline Red Thumb & 49.3 & 82.2 & 32.94 & 65.89 & 5.437 \\
\hline Smart & 50.5 & 82.65 & 32.2 & 66.63 & 4.605 \\
\hline \multicolumn{6}{|l|}{$\underline{\text { Reheated }}$} \\
\hline Adora & 49.14 & 81.27 & 32.14 & 62.47 & 5.807 \\
\hline Yellow Star & 49.38 & 82.52 & 33.14 & 65.04 & 4.896 \\
\hline Carlingford & 49.29 & 83.55 & 34.26 & 65.43 & 4.927 \\
\hline Purple Fiesta & 47.89 & 82.28 & 34.39 & 64.95 & 4.985 \\
\hline French Fingerlings & 49.44 & 82.99 & 33.55 & 64.6 & 5.13 \\
\hline Ciklamen & 49.04 & 83.13 & 34.09 & 66.95 & 4.874 \\
\hline Red Thumb & 49.02 & 86 & 36.98 & 64.06 & 4.59 \\
\hline Smart & 49.7 & 84.76 & 35.06 & 64.33 & 4.239 \\
\hline \multicolumn{6}{|l|}{ Baked } \\
\hline Adora & 50.4 & 81.5 & 31.1 & 63.37 & 5.02 \\
\hline Yellow Star & 50.24 & 83.2 & 33 & 64.05 & 5.1 \\
\hline Carlingford & 50.44 & 84.7 & 34.2 & 63.68 & 4.96 \\
\hline Purple Fiesta & 49.08 & 83.1 & 34 & 64.83 & 4.24 \\
\hline French Fingerlings & 49.71 & 82.8 & 33.1 & 64.32 & 4.66 \\
\hline Ciklamen & 50.08 & 81 & 30.9 & 64.28 & 5 \\
\hline Red Thumb & 50.53 & 81 & 30.4 & 64.08 & 4.49 \\
\hline Smart & 52.96 & 80.7 & 27.7 & 62.49 & 4.06 \\
\hline \multicolumn{6}{|l|}{ Microwaved } \\
\hline Adora & 52.7 & 80.7 & 28 & 63 & 3.18 \\
\hline Yellow Star & 52.2 & 80.6 & 28.5 & 63.4 & 4.96 \\
\hline Carlingford & 53.6 & 81.1 & 27.4 & 64.5 & 3.2 \\
\hline Purple Fiesta & 52.2 & 80.7 & 28.5 & 63.6 & 4.74 \\
\hline French Fingerlings & 53.4 & 80.1 & 26.7 & 63.7 & 3.6 \\
\hline Ciklamen & 52.5 & 80.4 & 27.9 & 63.6 & 4.77 \\
\hline Red Thumb & 53.8 & 79.9 & 26.1 & 69.7 & 3.48 \\
\hline Smart & 53.9 & 84.5 & 30.6 & 74.9 & 3.82 \\
\hline
\end{tabular}

All experiment data are the means of duplicate samples. 


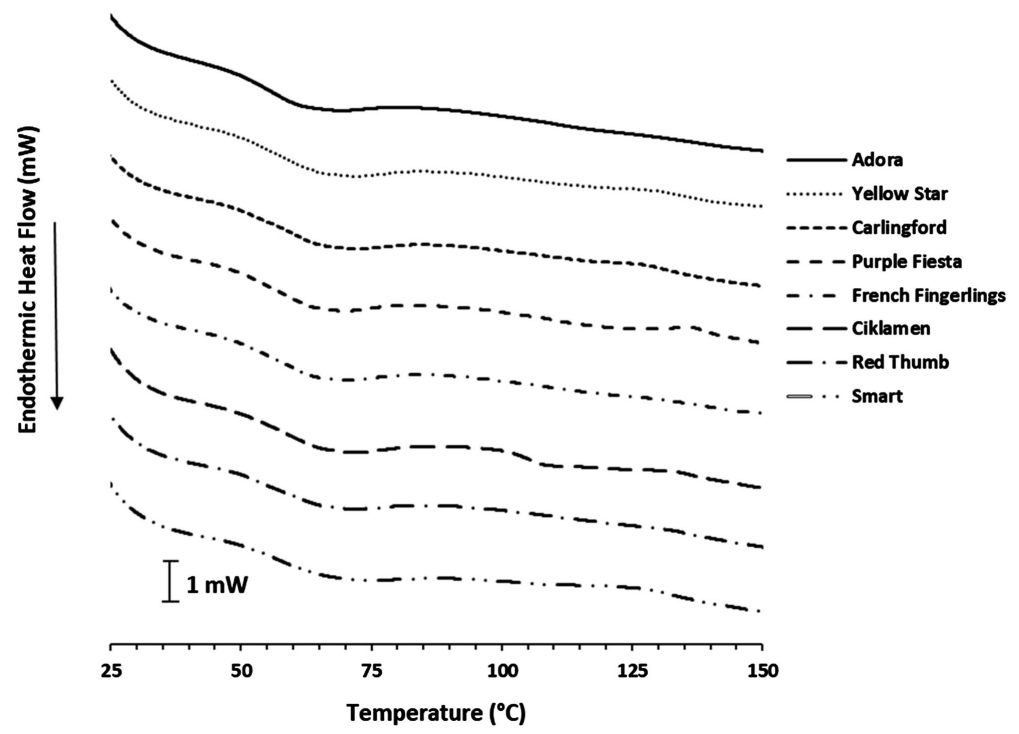

(a)

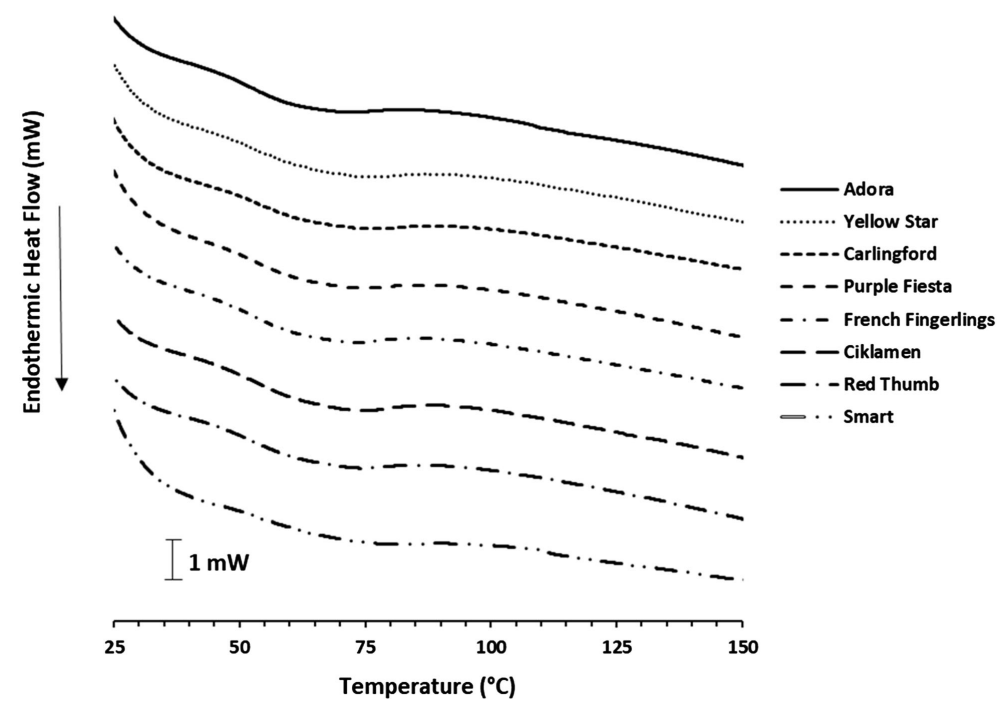

(b)

Figure 2. Thermograms of freeze dried retrograded potato samples after initial heating (a) and reheating in the DSC following 2 -weeks storage at $5^{\circ} \mathrm{C}(\mathrm{b})$.

and/or some new order structure was formed after processing. It also indicates that the gelatinization of those starches, which occurred during the processing and/or disruption of newly formed order structure, had taken place during the initial heating in the DSC. The enthalpy of this endothermic transition was found to be between 3.2 and $6.2 \mathrm{~J} / \mathrm{g}$ based on the mass of the dry matter (Table 1). The enthalpy was much lower than that of unprocessed potato dry matter, which has been determined to be $12-14 \mathrm{~J} / \mathrm{g}$ from our previous study [22], indicating that starch was either not fully gelatinized during this processing or it was fully gelatinized but formed a new order structure after processing. Onset temperature ranged from $49^{\circ} \mathrm{C}$ to $53.9^{\circ} \mathrm{C}$ and showed significant differences among varieties and processing methods. Retrograded and reheated samples had a sim- 
ilar $T_{o}$ while it increased slightly in baked and was highest in microwaved samples (Table $1, \mathrm{p} \leq 0.05$ ). Purple Fiesta showed the lowest $T_{o}$ whereas the highest was observed in Smart, irrespective of the processing method. $T_{p}$ and $T_{c}$ ranged from $62^{\circ} \mathrm{C}$ to $74.9^{\circ} \mathrm{C}$ and $80.4^{\circ} \mathrm{C}$ to $84.8^{\circ} \mathrm{C}$, respectively. Smart also showed highest $T_{c}$ and $T_{p}$, with the only exception being in baked potatoes. The enthalpy ranged from 3.2 to $6.2 \mathrm{~J} / \mathrm{g}$. The Smart variety also showed the lowest enthalpy in all processing methods, except for microwaved samples. Higher thermal transition temperatures and enthalpy are thought to result from a higher order degree of crystalline or double helical structure.

After initial heating and storage at low temperature, the gelatinized starch in potato usually re-associates and forms a new ordered structure during storage and this can contribute to the enzyme resistance of starch [14]. The thermograms (Figure 2(b)) of potato dry matter exhibited a single endotherm transition. The period of this specific phase is about 4 minutes by calculating the difference of end temperature and onset temperature then dividing the heat rate. This specific phase indicates the disruption of newly formed starch structure during two week storage (starch retrogradation) after initial heating for the potato dry matter in the presence of excess water. Significant differences were observed for $T_{o}, T_{c}$ and enthalpy of stored potato dry matters after initial heating (Table 2, $\mathrm{p} \leq 0.05$, Figure 2(b)). This further indicated that the structure of starch is different among these different potato dry matters. The order of $T_{o}$ was microwaved $>$ baked $>$ retrograded $>$ reheated samples. The highest $T_{o}$ was observed in the Smart variety. Similar to initial heating (starch gelatinization), the enthalpy of starch retrogradation was lowest in Smart.

\subsection{Starch Digestibility Characteristics}

Starch, its composition, and digestibility characteristics, such as RDS, SDS and RS, are important factors that affect GI and the glycemic impact. Consequently, TS and the nutritionally important starch fractions (RDS, SDS and RS) were analyzed. The TS content in potato dry matter varied from $54 \%$ to $69 \%$. Significant differences were observed in TS between varieties and processing methods (Figure 3, $\mathrm{p} \leq 0.05$ ). The lowest TS was obtained in Smart for all processing methods, significantly so with respect to the other varieties, followed by Yellow Star and Carlingford. TS was lowest in retrograded and reheated Smart potatoes and was similar to retrograded and baked, but significantly lower than microwaved Smart. In general, Purple Fiesta registered the highest TS. This result shows that there is an inverse relation between TS and moisture content given that highest moisture and lowest TS were observed in Smart, Yellow Star and Carlingford whereas Purple Fiesta had the lowest moisture and highest TS contents. The low TS and high moisture content may have also had a desirable effect on lowering the GI, as already highlighted in our earlier studies [20]. The amylose content varied between varieties and processing methods and ranged from $22 \%$ to $33 \%$, which was similar to reported values in potatoes [10] (Figure 4, $\mathrm{p} \leq 0.05$ ). 
Table 2. Retrogradation characteristics of potato dry matter.

\begin{tabular}{|c|c|c|c|c|c|}
\hline Cooking/Variety & $\begin{array}{c}\text { Onset } \\
\text { temperature } \\
T_{o}\left({ }^{\circ} \mathrm{C}\right)\end{array}$ & $\begin{array}{c}\text { Conclusion } \\
\text { temperature } \\
T_{c}\left({ }^{\circ} \mathrm{C}\right)\end{array}$ & $\begin{array}{c}T_{c}-T_{o} \\
\left({ }^{\circ} \mathrm{C}\right)\end{array}$ & $\begin{array}{c}\text { Peak } \\
\text { temperature } \\
T_{p}\left({ }^{\circ} \mathrm{C}\right)\end{array}$ & $\begin{array}{l}\text { Enthalpy } \\
\Delta \mathrm{H}\left(\mathrm{J} \cdot \mathrm{g}^{-1}\right)\end{array}$ \\
\hline \multicolumn{6}{|l|}{ Retrograded } \\
\hline Adora & 46 & 82.1 & 36.1 & 62.5 & 6.09 \\
\hline Yellow Star & 46.5 & 85.2 & 38.7 & 66.4 & 5.86 \\
\hline Carlingford & 46.9 & 84.6 & 37.8 & 64.2 & 5.42 \\
\hline Purple Fiesta & 46 & 83.2 & 37.2 & 64.3 & 6.19 \\
\hline French Fingerlings & 45.9 & 83.2 & 37.3 & 63.5 & 6.85 \\
\hline Ciklamen & 45.9 & 84.5 & 38.7 & 67.2 & 7.21 \\
\hline Red Thumb & 45.7 & 84.9 & 39.2 & 65.2 & 6.34 \\
\hline Smart & 47.7 & 82 & 34.3 & 65.7 & 4.19 \\
\hline \multicolumn{6}{|l|}{ Reheated } \\
\hline Adora & 46.2 & 83.9 & 37.7 & 64.2 & 6.14 \\
\hline Yellow Star & 46.5 & 81.3 & 34.8 & 65.8 & 6.05 \\
\hline Carlingford & 45.1 & 78.8 & 33.7 & 65.8 & 5.46 \\
\hline Purple Fiesta & 46.6 & 78.4 & 31.7 & 65.5 & 6.02 \\
\hline French Fingerlings & 44.4 & 78.6 & 34.1 & 65.3 & 6.05 \\
\hline Ciklamen & 45.2 & 81.7 & 36.5 & 67.4 & 6.14 \\
\hline Red Thumb & 44.3 & 85.4 & 41.1 & 64.5 & 6.19 \\
\hline Smart & 46.8 & 81.8 & 35 & 66 & 3.91 \\
\hline \multicolumn{6}{|l|}{ Baked } \\
\hline Adora & 46.3 & 84.4 & 38.1 & 64 & 6.31 \\
\hline Yellow Star & 46 & 85.1 & 39.1 & 66.3 & 5.99 \\
\hline Carlingford & 46.2 & 84.5 & 38.2 & 63.6 & 6.01 \\
\hline Purple Fiesta & 46.2 & 84.6 & 38.4 & 65.2 & 6.15 \\
\hline French Fingerlings & 45.9 & 83.9 & 38.1 & 65.3 & 6.13 \\
\hline Ciklamen & 45.9 & 84.2 & 38.3 & 64.2 & 6.36 \\
\hline Red Thumb & 45.6 & 84.7 & 39.1 & 66.1 & 6.44 \\
\hline Smart & 50.8 & 81.3 & 30.6 & 64.3 & 4.52 \\
\hline \multicolumn{6}{|l|}{ Microwaved } \\
\hline Adora & 48.3 & 82.5 & 34.2 & 62.9 & 6.49 \\
\hline Yellow Star & 49.6 & 81.7 & 32.1 & 65.4 & 5.28 \\
\hline Carlingford & 50 & 81.9 & 31.9 & 64.2 & 4.99 \\
\hline Purple Fiesta & 49.5 & 82.3 & 32.7 & 64.7 & 5.35 \\
\hline French Fingerlings & 50.2 & 82.1 & 31.8 & 64.1 & 5.54 \\
\hline Ciklamen & 49.7 & 83 & 33.3 & 66.1 & 5.56 \\
\hline Red Thumb & 49.1 & 82.5 & 33.4 & 64.9 & 5.73 \\
\hline Smart & 51.7 & 81.4 & 29.7 & 62.8 & 3.63 \\
\hline
\end{tabular}

All experiment data are the means of duplicate samples. 


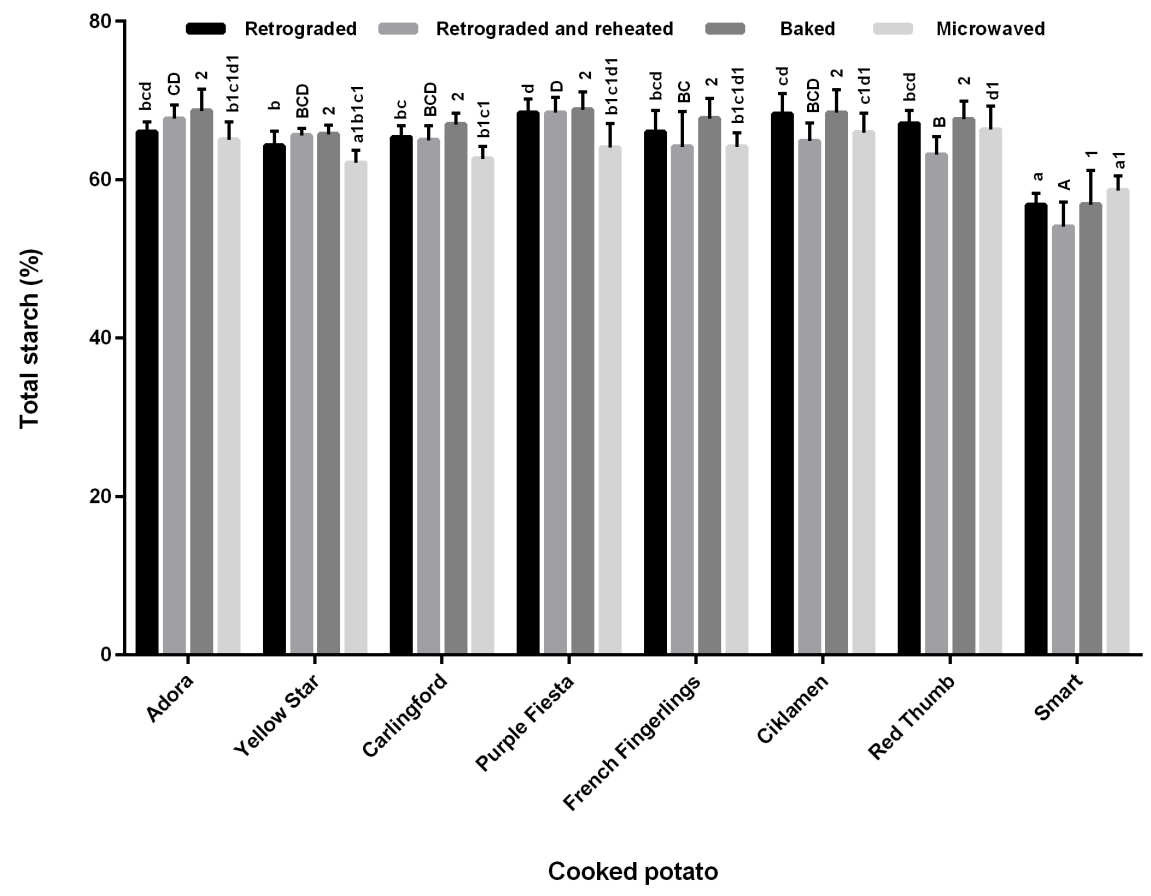

Figure 3. Effect of variety and processing on total starch contents of potatoes. Error bars represent standard error from two experiments $(n=4)$. Bars with different letters, a - d, A - D, 1 - 2 and a1 - d, denote significant differences between varieties when performing the same processing method (Tukey's test, $\mathrm{p} \leq 0.05$ ).

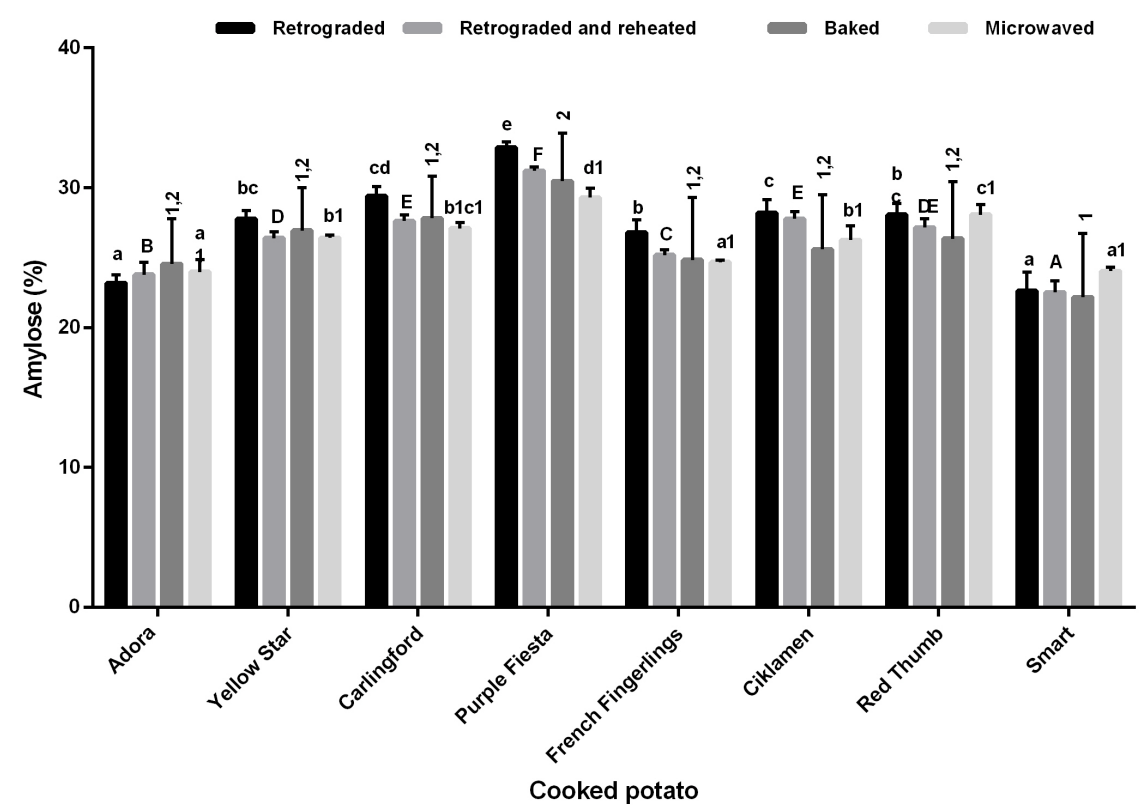

Figure 4. Effect of variety and processing on amylose contents of potatoes. Error bars represent standard error from two experiments $(n=4)$. Bars with different letters, a - d, A - F, 1 - 2 and a1 - d1, denote significant differences between varieties when performing the same processing method (Tukey's test, $\mathrm{p} \leq 0.05$ ).

In general, Smart and Adora had the lowest amylose content whereas the highest amylose content was obtained in Purple Fiesta for all processing methods. It is 
also noticeable that Smart had the lowest total starch which could explain the lowest amylose content in it. Higher variability was observed in the contents of baked potatoes. The amylose content was higher in retrograded potatoes in all varieties, except Smart and Adora. A greater percentage of amylose has been generally linked to a higher tendency of retrogradation and hence will slow down digestion of starch and rapid spike in blood glucose levels [19]. It has been reported that genotype, growing conditions and processing methods affect the amylose content in potatoes [19] [20].

Among the digestible fractions of starch, only RDS showed significant differences between varieties and for some processing methods. RDS varied from 34\% to $54 \%$. The lowest RDS was observed in Smart for all processing methods followed by Carlingford, Yellow Star, Ciklamen and Red Thumb (Figure 5, p $\leq$ 0.05). Adora had the highest RDS and was similar to Purple Fiesta and French Fingerlings. No significant difference was observed among the various processing methods in Carlingford, Yellow Star, Purple Fiesta, Red Thumb, French Fingerlings, Cikalmen and Adora. RDS has greater influence on adversely affecting the GI by contributing sudden changes in blood sugar levels. Even though, no significant difference was observed for SDS ranging from $4 \%$ to $13 \%$, between varieties and processing methods, the highest SDS contents were observed in Smart, Purple Fiesta, and Yellow Star. In general, retrograded and reheated potato varieties contained higher SDS compared to microwaved and baked methods, irrespective of the variety (data not shown). Higher RS was obtained for baked and microwaved potatoes compared to retrograded and reheated samples (Figure 6). Baked Purple Fiesta had significantly higher RS and superior to all varieties except Yellow Star, Carlingford, French Fingerlings ( $\mathrm{p} \leq 0.05)$. Among microwaved potatoes, the highest RS was obtained in Red Thumb and was similar to Smart and Yellow Star, but superior to Adora, Carlingford, Purple Fiesta, French Fingerlings and Ciklamen. No significant difference was observed in RS

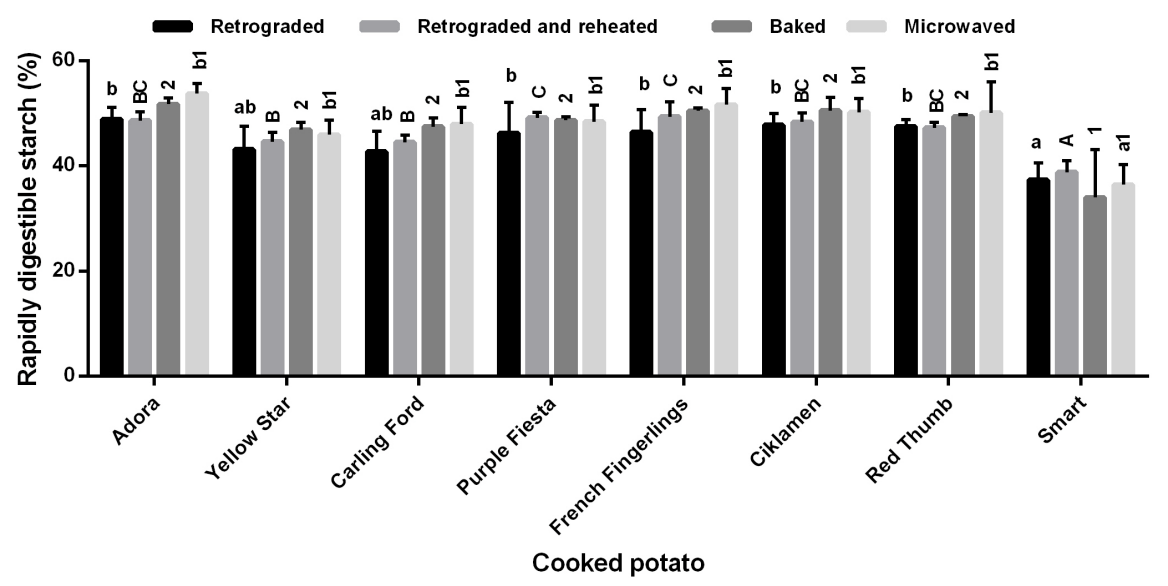

Figure 5. Effect of variety and processing on rapidly digestible starch contents of potatoes. Error bars represent standard error from two experiments $(n=4)$. Bars with different letters, $a-b, A-C, 1-2$ and $a 1-b 1$, denote significant differences between varieties when performing the same processing method (Tukey's test, $\mathrm{p} \leq 0.05$ ). 


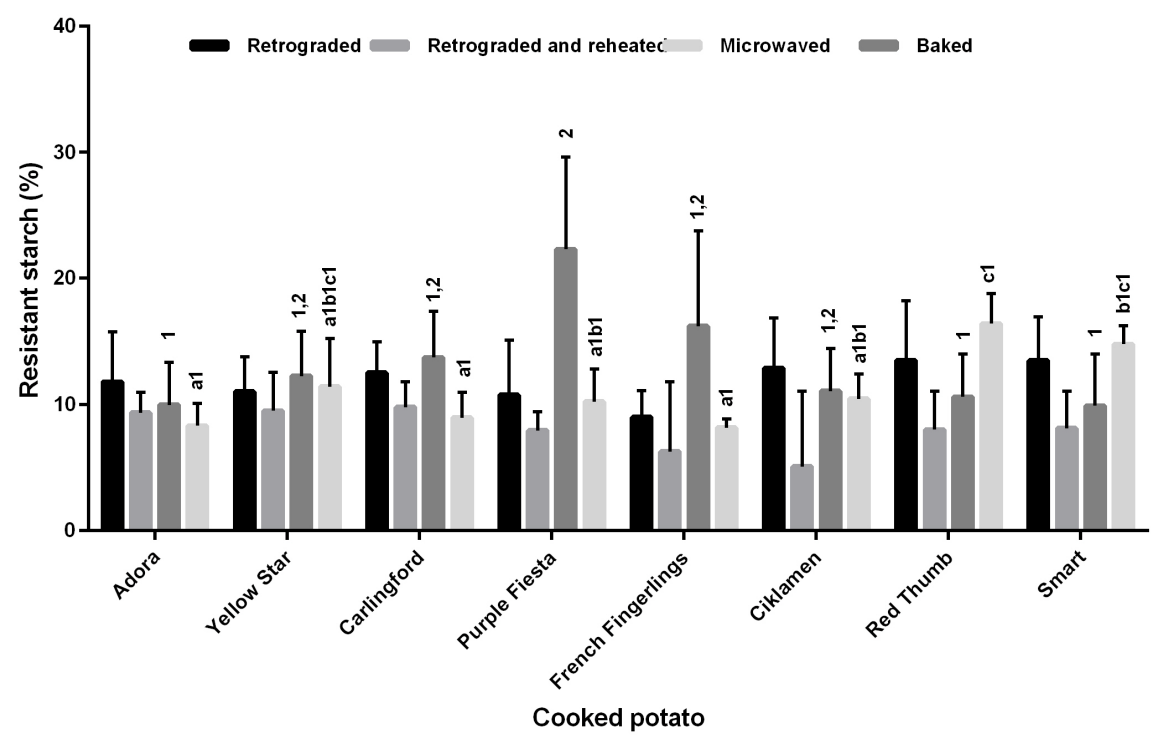

Figure 6. Effect of variety and processing on the resistant starch content of potatoes. Error bars represent standard error from two experiments $(n=4)$. Bars with different letters, $1-2$ and a1 - cl, denote significant differences between varieties when performing the same processing method (Tukey's test, $\mathrm{p} \leq 0.05$ ).

contents between retrograded and reheated varieties. However, Smart and Red Thumb had the highest RS in retrograded processing followed by Ciklamen, Carlingford, Adora, Yellow Star, Purple Fiesta and French Fingerlings. RS was higher in retrograded, baked and microwaved potatoes compared to SDS in these processing methods.

\subsection{Estimated Glycemic Index and Glycemic Load}

Estimated glycemic index (eGI) varied significantly between varieties and processing methods (Figure 7, p $\leq 0.05$ ). Smart registered the lowest eGI for all processing methods while all varieties, except for Smart, had the same eGI in retrograded, reheated and baked potatoes. For microwaved samples, Smart had the lowest eGI and the highest eGI was observed in Ciklamen, French Fingerlings, and Adora. A similar eGI was obtained in microwaved Red Thumb, Yellow Star, Carlingford and Purple Fiesta. Among all processing methods, retrograded potatoes registered the lowest eGI, except for microwaved Smart. Our earlier study also showed that the lowest eGI is observed in retrograded potatoes [20]. The fact that the lowest RDS was observed in Smart, for all processing methods, may have contributed to this being the lowest observed eGI as RDS has strong influence in determining eGI [20] [26] [27]. The susceptibility of processed starch to enzymatic digestion is determined largely by the extent of starch structure disruption that occurs during gelatinization as well as aggregates that form during the subsequent retrogradation [28]. A similar result, which highlighted this lower eGI in retrograded potatoes, was obtained by Moreira \& Wolever [26]. The higher transition temperature of Smart potatoes suggests that crystalline regions of low eGI potatoes are more stable than those of other varieties. Moreover, 


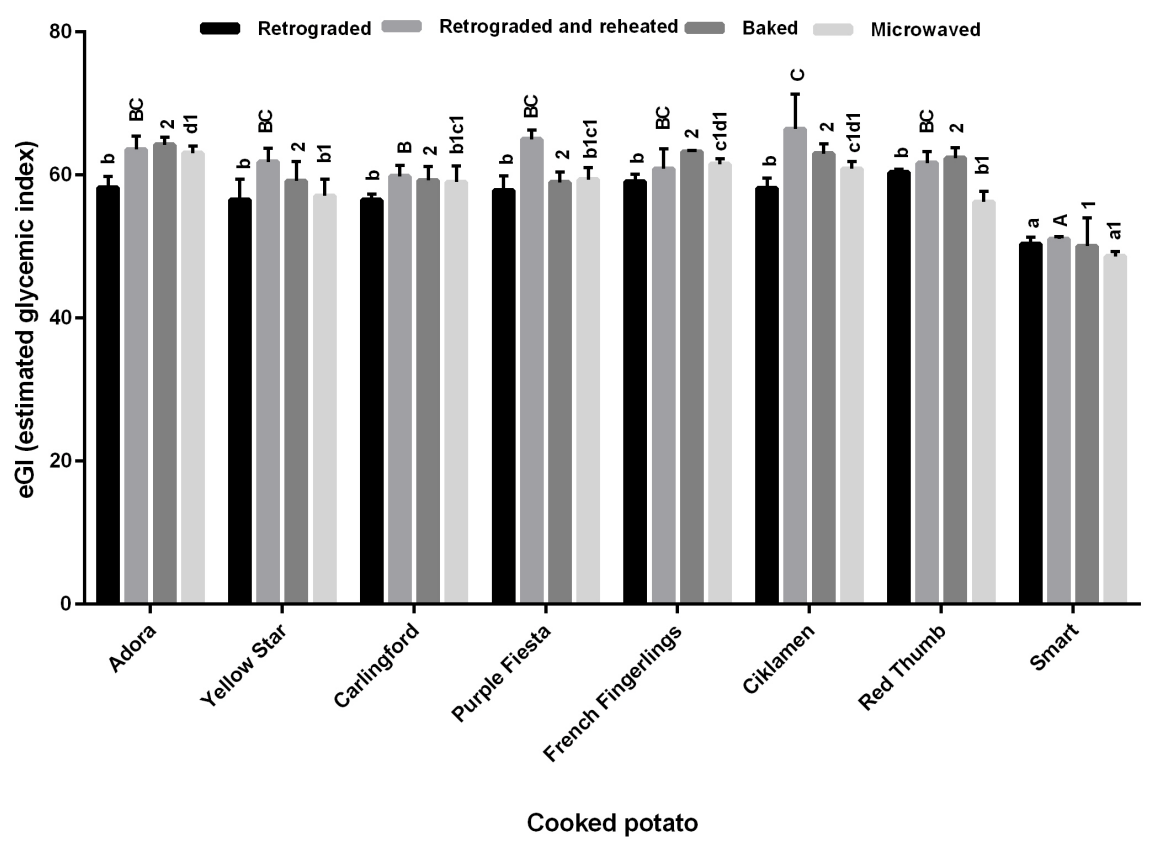

Figure 7. Effect of variety and processing on the estimated glycemic index of potatoes. Error bars represent standard error from two experiments $(n=4)$. Bars with different letters, $a-b, A-C, 1-2$ and a1 - d1, denote significant differences between varieties within the same processing method (Tukey's test, $\mathrm{p} \leq 0.05$ ).

different processing methods as well as starch characteristics and microstructure influence the GI of potatoes and potato products [19] [29] [30]. Estimated glycemic load (eGL) varied from 3 to 10 and significant differences were observed between varieties and processing methods (Figure 8, $\mathrm{p} \leq 0.05$ ). According to GL classification, a food product with GL values higher than 20 is considered as high, between 11 - 19 as medium, and less than 10 is considered as a low glycemic food [9]. Hence, all the varieties tested fell within the low GL category. Similar to RDS and eGI, the lowest eGL was obtained in Smart potatoes with all processing methods, ranging from 3.2 in retrograded to 4.4 in baked potatoes. Retrograded potatoes showed the lowest eGL in all varieties and this was expected given that the eGI was lowest in retrograded potatoes. In retrograded potatoes, Smart showed the lowest eGI, followed by Carlingford, Yellow Star, Adora, French Fingerlings, Red Thumb, Ciklamen and Purple Fiesta. Purple Fiesta registered the highest eGL in reheated samples followed by Ciklamen, Red Thumb, Adora, and French Fingerlings. Ciklamen, Red Thumb, Adora and French Fingerlings had the same eGL in reheated samples. A more or less similar trend was noted for eGL of retrograded potato varieties, being observed in reheated, baked and microwaved potato varieties. In general, baked potato samples had a significantly higher eGL in all varieties compared to other processing methods.

The Pearson correlation studies between eGI and RDS, SDS, RS, total moisture, total starch, and amylose, for potato samples treated with the various processing methods were carried out to investigate the relationships between GI 


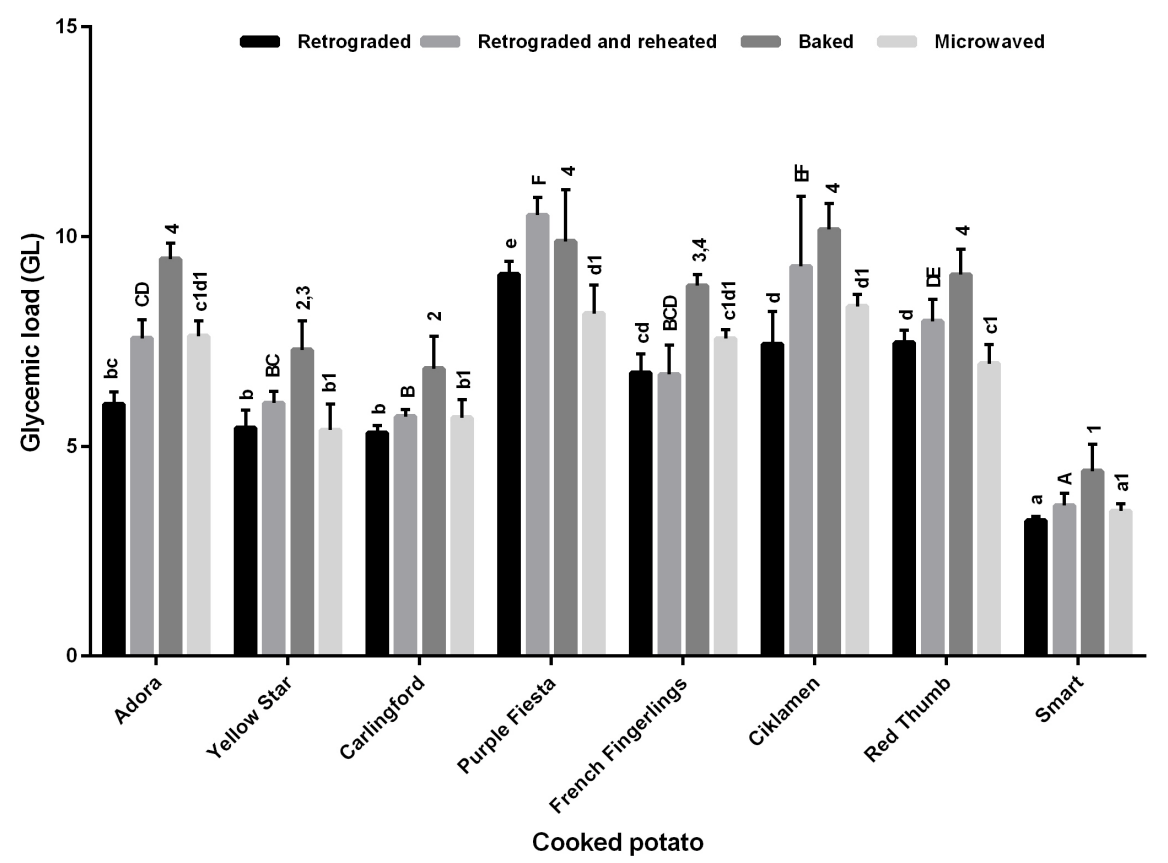

Figure 8. Effect of variety and processing on glycemic load of potatoes. Error bars represent standard error from two experiments $(n=2)$. Bars with different letters, $a-b, A$ - C, 1 - 2 and a1 - d1, denote significant differences between varieties when performing the same processing method (Tukey's test, $\mathrm{p} \leq 0.05$ ).

and the above variables. A significant negative correlation was observed between eGI and moisture for all processing methods, except in the case of retrograded samples (Table 3, $\mathrm{p} \leq 0.05$ ). A significant positive correlation was observed for RDS in all processing methods and the correlation was very high in reheated (0.84), baked (0.79) and microwaved (0.74) samples. Clinical studies conducted by Moreira and Wolever [26], and in an animal study by Sasaki, Sotome, and Okadome [26], also reported significant positive correlations between GI and RDS for various potato varieties. The present study demonstrates that the estimation of GI using in vitro digestion resulted in similar observations to those shown in clinical trials and that the estimation of GI in vitro can be used as a less expensive tool with respect to clinical trials for the purpose of assessing the GI of potatoes. Ek, Wang, Copeland, \& Brand-Miller [6] also noted that the GI values calculated from clinical trials were also strongly and positively correlated with the percentage of in vitro enzymatic hydrolysis of starch in cooked potatoes, thus validating the use of in vitro methods for estimating GI. Similar results were also observed in our earlier studies, highlighting this positive correlation between eGI and RDS [20]. From our earlier study, Pinhero et al. [20] showed a positive correlation between eGI and RDS ranging from 0.5 to 0.99 among 14 retrograded potato samples. However, in this study a low positive correlation of 0.43 was observed. Here we calculated the correlation pooling the eight varieties together whereas in the previous study positive correlation was studied for individual variety. The positive correlation between eGI and RDS for reheated, baked and microwaved varieties was $0.84,0.79$ and 0.74 , respectively. A negative 
Table 3. Pearson correlation coefficients of the relationship among various starch parables and estimated glycemic index.

\begin{tabular}{cccccccc}
\hline Processing & Coefficient & Moisture & Amylose & Total Starch & RDS & SDS & RS \\
\hline \multirow{2}{*}{ Retrograded } & $\mathrm{r}$ & -0.42 & -0.25 & 0.02 & $0.43^{*}$ & 0.11 & -0.01 \\
& $\mathrm{p}(2$ tailed $)$ & $\mathrm{NS}$ & $\mathrm{NS}$ & $\mathrm{NS}$ & 0.017 & $\mathrm{NS}$ & $\mathrm{NS}$ \\
\multirow{2}{*}{ Reheated } & $\mathrm{r}$ & -0.74 & -0.09 & -0.34 & $0.84^{* * *}$ & -0.38 & -0.39 \\
& $\mathrm{p}(2$ tailed $)$ & $<0.001$ & $\mathrm{NS}$ & $\mathrm{NS}$ & $<0.0001$ & 0.03 & 0.02 \\
\multirow{2}{*}{ Baked } & $\mathrm{r}$ & -0.58 & -0.01 & 0.28 & $0.79^{* * *}$ & -0.27 & -0.13 \\
& $\mathrm{p}(2$ tailed $)$ & 0.019 & $\mathrm{NS}$ & $\mathrm{NS}$ & $<0.001$ & $\mathrm{NS}$ & $\mathrm{NS}$ \\
& $\mathrm{r}$ & -0.51 & -0.55 & 0.15 & $0.74^{* * *}$ & -0.33 & -0.37 \\
Microwaved & $\mathrm{p}(2$ tailed $)$ & 0.043 & 0.001 & $\mathrm{NS}$ & $<0.0001$ & $\mathrm{NS}$ & 0.037 \\
\hline
\end{tabular}

Significant correlations are denoted by ${ }^{*}{ }^{* * *}$.

correlation was observed for SDS only in the case of reheated samples and for RS in reheated and microwaved samples. We observed similar results with SDS, with the exception of RS of retrograded and baked potatoes [20]. With regard to amylose, a negative correlation was observed only for microwaved samples. In general, no correlation was observed for total starch and eGI which also did not show correlation between total starch and eGI [20]. It is suggested that total starch may not be the single factor contributing to eGI as the various fractions such as RDS, SDS and RS have greater influence. A higher SDS and RS as well as lower RDS contents can reduce the impact of higher total starch on eGI. Similar results for eGI and total starch and amylose were reported in cooked potatoes [6] [20]. It has been previously reported that [19] [20], early maturing potato varieties usually have a lower dry matter, higher moisture content and different starch structure. This study also corroborated the previous findings. The examination of 8 varieties with various processing methods indicated that RDS had a stronger positive relationship while moisture and amylose negatively influenced the eGI, thus impacting the glycemic response.

\section{Conclusion}

Evaluating the nutritional profile of starch in eight potato varieties, using four processing methods, showed that varieties differed in terms of their moisture content, total starch, amylose, starch digestibility, eGI, and eGL. Smart potatoes had the lowest total starch, amylose, RDS, eGI and eGL and highest moisture contents for all processing methods. Smart is classified as a low eGI food while Adora, Yellow Star, Carlingford, Purple Fiesta, French Fingerlings, Ciklamen, Red Thumb are classified as medium eGI. A significant and strong positive correlation was observed between eGI and RDS, whereas a significant and strong negative correlation was observed between eGI and moisture. The eGL values of all the potato varieties tested fell between 3 and 10; thus it can be considered as low GL foods. Even though the eGI ranged from low to medium, all the potato varieties tested show low eGL. We also showed that foods classified as high GI 
may not be the sole criteria to choose foods for the management of diabetes or weight loss, but GL should also be taken into account. The present study further corroborated RDS as a major starch factor contributing to eGI. In summary, our results demonstrated that variety, processing methods, and the starch composition influences the glycemic impact.

\section{Acknowledgements}

The authors acknowledge technical help from Vanessa Currie, Plant Agriculture and Elizabeth Donner from Food Research Centre, AAFC, Guelph was also greatly appreciated.

\section{Funding}

This work was supported by the Ontario Ministry of Agriculture, Food and Rural Affairs and the Potato Cluster 11 of the Agri Innovation Program of Agriculture and Agri-Food Canada. The support of the Natural Sciences and Engineering Research Council of Canada is also gratefully acknowledged.

\section{Conflicts of Interest}

The authors declare no conflicts of interest regarding the publication of this paper.

\section{References}

[1] Camire, M.E., Kubow, S. and Donnelly, D.J. (2009) Potatoes and Human Health. Critical Reviews in Food Science and Nutrition, 49, 823-840.

[2] Wolever, T.M.S. (2006) The Glycemic Index: A Physiological Classification of Dietary Carbohydrates. CABI Publishing, Wallingford, UK, 120-123. https://doi.org/10.1079/9781845930516.0000

[3] Van Dam, R.M. and Seidell, J.C. (2007) Carbohydrate Intake and Obesity. European Journal of Clinical Nutrition, 61, S75-S99. https://doi.org/10.1038/sj.ejcn.1602939

[4] Foster-Powell, K., Holt, S.H. and Brand-Miller, J.C. (2002) International Tables of Glycemic Index and Glycemic Load Values: 2002. The American Journal of Clinical Nutrition, 76, 5-56. https://doi.org/10.1093/ajcn/76.1.5

[5] Atkinson, F.S., Foster-Powell, K. and Brand-Miller, J.C. (2008) International Tables of Glycemic Index and Glycemic Load Values. Diabetic Care, 3, 2281-2283. https://doi.org/10.2337/dc08-1239

[6] Ek, K.L., Wang, S., Copeland, L. and Brand-Miller, J.C. (2014) Discovery of a Low-Glycaemic Index Potato and Relationship with Starch Digestion in Vitro. British Journal of Nutrition, 111, 699-705. https://doi.org/10.1017/S0007114513003048

[7] Salmeron, J., Monson, J.E., Stampfer, M.S., Colditz, G.A., Wing, A.L. and Willett, W.C. (1997) Dietary Fiber, Glycemic Load and Risk of Non-Insulin Dependent Diabetes Mellitus on Woman. The Journal of American Medical Association, 277, 472-477. https://doi.org/10.1001/jama.1997.03540300040031

[8] Brand-Miller, J.C. (2003) Glycemic Load and Chronic Diseases. Nutrition Reviews, 61, S49-S55. https://doi.org/10.1301/nr.2003.may.S49-S55

[9] Brennan, C.S. (2005) Dietary Fiber, Glycemic Response and Diabetes. Molecular 
Nutrition and Food Research, 49, 560-570. https://doi.org/10.1002/mnfr.200500025

[10] Pérez, S. and Bertoft, E. (2010) The Molecular Structures of Starch Components and Their Contribution to the Architecture of Starch Granules: A Comprehensive Review. Starch, 62, 389-420. https://doi.org/10.1002/star.201000013

[11] Hoover, R. (2001) Composition, Molecular Structure, and Physico-Chemical Properties of Tuber and Root Starches: A Review. Carbohydrate Polymers, 45, 253-267. https://doi.org/10.1016/S0144-8617(00)00260-5

[12] Bertoft, E. and Blennow, A. (2008) Structure of Potato Starch. In: Singh, J. and Kaur, L., Eds., Advances in Potato Chemistry and Technology, Elsevier, Oxford, 83-98. https://doi.org/10.1016/B978-0-12-374349-7.00004-0

[13] Behall, K.M., Scholfield, D.J. and Canary, J. (1988) Effect of Starch Structure on Glucose and Insulin Response in Adults. American Journal of Clinical Nutrition, 47, 428-432. https://doi.org/10.1093/ajcn/47.3.428

[14] Englyst, H.N., Kingman, S.M. and Cummings, J.H. (1992) Classification and Measurement of Nutritionally Important Starch Fractions. European Journal of Clinical Nutrition, 46, S33-S50.

[15] Birt, D.F., Boylston, T., Hendrich, S., Jane, J.L., Hollis, J., Li, L., Mcclelland, J., Moore, S., Phillips, G.J., Rowling, M., Schalinske, K., Scott, M.P. and Whitley, E.M. (2013) Resistant Starch: Promise for Improving Human Health. Advances in Nutrition, 4, 587-601. https://doi.org/10.3945/an.113.004325

[16] Englyst, K.N., Vinoy, S., Englyst, H.N. and Lang, V. (2003) Glycemic Index of Cereal Products Explained by Their Content of Rapidly and Slowly Available Glucose. British Journal of Nutrition, 89, 329-339.https://doi.org/10.1079/BJN2002786

[17] Lynch, D.R., Liu, Q., Tarn, T.R., Bizimungu, B., Chen, Q., Harris, P., Chik, C.L. and Skjodt, N.M. (2007) Glycemic Index-A Review and Implications for the Potato Industry. American Journal of Potato Research, 84, 179-190. https://doi.org/10.1007/BF02987141

[18] Fernandes, G., Velangi, A. and Wolever, T.M.S. (2005) Glycemic Index of Potatoes Commonly Consumed in North America. Journal of the American Dietetic Association, 105, 557-562. https://doi.org/10.1016/j.jada.2005.01.003

[19] Nayak, B., Berrios, J.J. and Tang, J (2014) Impact of Food Processing on the Glycemic Index (GI) of Potato Products. Food Research International, 56, 35-46. https://doi.org/10.1016/j.foodres.2013.12.020

[20] Pinhero, R.G., Waduge, R.N., Liu, Q., Sullivan, J. A., Tsao, R., Bizimungu, B. and Yada, R.Y. (2016) Evaluation of Nutritional Profiles of Starch and Dry Matter from Early Potato Varieties and Its Estimated Glycemic Impact. Food Chemistry, 203, 356-366. https://doi.org/10.1016/j.foodchem.2016.02.040

[21] Williams, P.C., Kuzina, F.D. and Hlynka, I. (1970) A Rapid Colorimetric Procedure for Estimating the Amylose Contents of Starches and Flours. Cereal Chemistry, 47, 411-420.

[22] Lu, Z.-H., Donner, E.A., Yada, R.Y. and Liu, Q. (2012) The Synergistic Effects of Amylose and Phosphorus on Rheological, Thermal and Nutritional Properties of Potato Starch and Gel. Food Chemistry, 133, 1214-1221. https://doi.org/10.1016/j.foodchem.2011.07.029

[23] Chung, H.J., Lim, H.S. and Lim, S.T (2006) Effect of Partial Gelatinization and Retrogradation on the Enzymatic Digestion of Waxy Rice Starch. Journal of Cereal Science, 43, 353-359.

[24] Goni, I., Garcia-Alonso, A. and Saura-Calixto, F. (1997) A Starch Hydrolysis Pro- 
cedure to Estimate Glycemic Index. Nutrition Research, 17, 427-437. https://doi.org/10.1016/S0271-5317(97)00010-9

[25] Granfeldt, Y. (1994) Food Factors Affecting Metabolic Responses to Cereal Products. Department of Applied Nutrition and Food Chemistry, Lund University, Lund.

[26] Moreira, T.S. and Wolever, T.M.S. (2011) The Role of Starch Physicochemical Properties in Determining the Glycemic Index of Potatoes. The FASEB Journal, 251, b1196.

[27] Sasaki, T., Sotome, I. and Okadome, H. (2015) In Vitro Starch Digestibility and in Vivo Glucose Response of Gelatinized Potato Starch in the Presence of Non-Starch Polysaccharides. Starch-Stärke, 67, 415-423. https://doi.org/10.1002/star.201400214

[28] Wang, S., Li, C., Copeland, L., Niu, Q. and Wang, S. (2015) Starch Retrogradation: A Comprehensive Review. Comprehensive Reviews in Food Science and Food Safety, 14, 568-585. https://doi.org/10.1111/1541-4337.12143

[29] Ciftci, S., Büyüktuncer Demirel, Z., Köksel, H. and Ozel, G. (2017) MON-P153: Does Different Preperation and Cooking Methods Impact on the Glycemic Index of the Potato? Clinical Nutrition, 36, S235. https://doi.org/10.1016/S0261-5614(17)30933-0

[30] Tian, J.H., Chen, S.G., Chen, J.C., Liu, D.H. and Ye, X.Q. (2018) Cooking Methods Altered the Microstructure and Digestibility of the Potato. Starch-Stärke, 70, Article ID: 1700241. https://doi.org/10.1002/star.201700241

\section{Abbreviations}

RDS, Rapidly Digestible Starch; SDS, Slowly Digestible Starch; RS, Resistant Starch; eGI, estimated Glycemic Index; eGL, estimated Glycemic Load 